University of Wollongong

Research Online

Australian Institute for Innovative Materials -

Papers

Australian Institute for Innovative Materials

$1-1-2005$

\title{
Effect of doping on flux pinning of GdBa2Cu307-y
}

L Zhang

Nanjing University, Iz811@uow.edu.au

$\mathrm{X} \mathrm{B} \mathrm{Xu}$

Nanjing University

S Y Ding

Nanjing University

M H Zheng

General Research Institute for Non-ferrous Metals

L Xiao

General Research Institute for Non-ferrous Metals

See next page for additional authors

Follow this and additional works at: https://ro.uow.edu.au/aiimpapers

Part of the Engineering Commons, and the Physical Sciences and Mathematics Commons

Research Online is the open access institutional repository for the University of Wollongong. For further information contact the UOW Library: research-pubs@uow.edu.au 


\title{
Effect of doping on flux pinning of GdBa2Cu307-y
}

\author{
Abstract \\ Cylindrical single grains of GdBa 2Cu30y sGd-123d with a diameter of $25 \mathrm{~mm}$ were successfully \\ fabricated by melt-texture growth sMTGd process in air to study the influence of different starting \\ powders on flux pinning. Measurements of the magnetic critical current density $s J c d$ showed that it was \\ possible to fabricate large Gd-123 single grain with a high $\mathrm{Jc}$ at high temperatures and fields by means of \\ properly controlling the starting powders of $\mathrm{Gd} 2 \mathrm{O} 3, \mathrm{BaCO} 3$, and $\mathrm{CuO}$ before the process. \\ Keywords \\ Effect, doping, flux, pinning, GdBa2Cu3O7 \\ Disciplines \\ Engineering | Physical Sciences and Mathematics

\section{Publication Details} \\ Zhang, L., Xu, X., Ding, S., Zheng, M., Xiao, L., Ren, H., Jiao, Y., Wang, X., Lin, Z. \& Zhu, J. (2005). Effect of \\ doping on flux pinning of GdBa2Cu307-y. Journal of Applied Physics, 97 (10), 10B101-1-10B101-3.

\section{Authors} \\ L Zhang, X B Xu, S Y Ding, M H Zheng, L Xiao, H T Ren, Y L Jiao, Xiaolin Wang, Zhi W. Lin, and Jian G. Zhu
}




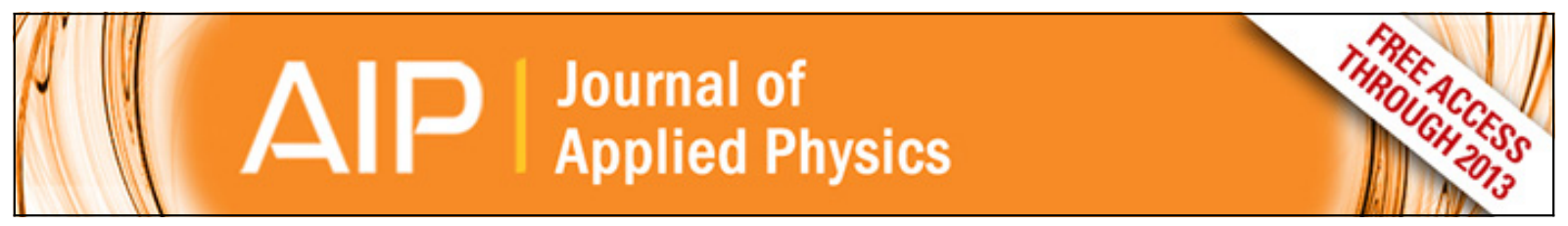

\section{Effect of doping on flux pinning of Gd Ba $2 \mathrm{Cu} 307 \mathrm{y}$}

L. Zhang, X. B. Xu, S. Y. Ding, M. H. Zheng, L. Xiao, H. T. Ren, Y. L. Jiao, X. L. Wang, Z. W. Lin, and J. G. Zhu

Citation: Journal of Applied Physics 97, 10B101 (2005); doi: 10.1063/1.1846571

View online: http://dx.doi.org/10.1063/1.1846571

View Table of Contents: http://scitation.aip.org/content/aip/journal/jap/97/10?ver=pdfcov

Published by the AIP Publishing

\section{AlP Re-register for Table of Content Alerts}

\section{Create a profile. \\ Sign up today!}




\title{
Effect of doping on flux pinning of $\mathrm{GdBa}_{2} \mathrm{Cu}_{3} \mathrm{O}_{7-y}$
}

\author{
L. Zhang, X. B. Xu, and S. Y. Ding ${ }^{\text {a) }}$ \\ National Laboratory of Solid State Microstructures, Department of Physics, Nanjing University, \\ Nanjing 210093, People's Republic of China
}

M. H. Zheng, L. Xiao, H. T. Ren, and Y. L. Jiao

General Research Institute for Non-ferrous Metals, Beijing 100088, People's Republic of China

X. L. Wang
Institute for Superconducting and Electronic Materials, University of Vollongong,
New South Wales 2522, Australia

Z. W. Lin and J. G. Zhu

University of Technology Sydney, New South Wales 2007, Australia

(Presented on 11 November 2004; published online 2 May 2005)

\begin{abstract}
Cylindrical single grains of $\mathrm{GdBa}_{2} \mathrm{Cu}_{3} \mathrm{O}_{y}(\mathrm{Gd}-123)$ with a diameter of $25 \mathrm{~mm}$ were successfully fabricated by melt-texture growth (MTG) process in air to study the influence of different starting powders on flux pinning. Measurements of the magnetic critical current density $\left(J_{c}\right)$ showed that it was possible to fabricate large Gd-123 single grain with a high $J_{c}$ at high temperatures and fields by means of properly controlling the starting powders of $\mathrm{Gd}_{2} \mathrm{O}_{3}, \mathrm{BaCO}_{3}$, and $\mathrm{CuO}$ before the MTG process. (C) 2005 American Institute of Physics. [DOI: 10.1063/1.1846571]
\end{abstract}

\section{INTRODUCTION}

Since the discovery of high-temperature superconductors (HTSCs), their engineering applications at liquid-nitrogen temperatures and at fields have drawn much more attention. The large scale applications of HTSC materials are crucially dependent on their critical current density $\left(J_{c}\right)$ and irreversibility field $\left(H_{\text {irr }}\right)$. Unfortunately, a sintering sample of HTSC is of weak links and thus of very low $J_{c}$ and $H_{\text {irr }}$. To eliminate the weak links, the so-called melt-texture growth (MTG) process has been developed, enhancing $J_{c}$ significantly. ${ }^{1}$ To further improve $J_{c}$ the MTG process has been improved and tremendous progress has been made. ${ }^{2-4} \mathrm{It}$ was pointed out that the high $J_{c}$ of the textured $\mathrm{YBa}_{2} \mathrm{Cu}_{3} \mathrm{O}_{7-y}$ (Y-123) system originated from homogenously dispersed fine $\mathrm{Y}_{2} \mathrm{BaCuO}_{z}$ (Y-211) particles. We note that the oxygen deficient clusters are effective to pin flux lines, especially at relatively low fields, and are responsible for the second peak effect in $J_{c}{ }^{5-7}$

An important progress on the MTG process for Y-123 materials is the substitution of $\mathrm{Y}$ by light rare earth (LRE: $\mathrm{Nd}, \mathrm{Sm}, \mathrm{Eu}$, and Gd) elements. Contrasting with Y-123 compounds, the LRE-Ba-Cu-O (LRE-123) HTSCs, have higher $J_{c}$ and $H_{\text {irr }}$. However, the LRE-123 systems easily form into a $\mathrm{LRE}_{1+x} \mathrm{Ba}_{2-x} \mathrm{Cu}_{3} \mathrm{O}_{y}$ solid solution, a secondary phase in LRE-123 due to LRE-Ba substitution. A small amount of $\mathrm{LRE}_{1+x} \mathrm{Ba}_{2-x} \mathrm{Cu}_{3} \mathrm{O}_{y}$ dispersed finely in LRE-123 is considered to be another effective flux-pinning center, leading to a high $J_{c}, H_{\text {irr }}$, and the second peak effect at high fields. ${ }^{8,9}$ Meanwhile, the $\mathrm{LRE}_{1+x} \mathrm{Ba}_{2-x} \mathrm{Cu}_{3} \mathrm{O}_{y}$ phase will result in a deterioration of superconductivity, lowering the $T_{c}$. To avoid excessive LRE-Ba substitution, variations of the MTG processes, such as postannealing in Ar atmosphere, using of Ba-

\footnotetext{
a) Author to whom correspondence should be addressed; electronic mail: syding@nju.edu.cn
}

rich precursors, and employing large crystallization rate, have been developed. ${ }^{10,11}$ In addition, some amount of the $\mathrm{LRE}_{2} \mathrm{BaCuO}_{5}$ (LRE-211) phase are added into the LRE-123 systems to improve the flux pinning. Because $\mathrm{GdBa}_{2} \mathrm{Cu}_{3} \mathrm{O}_{7-y}$ (Gd-123) in the LRE-123 systems shows the slightest $\mathrm{Gd}-\mathrm{Ba}$ substitution even when fabricated in air, and the "in air" processing will lower fabricating cost, the process of fabricating Gd-123 in air has received much more interests. ${ }^{12-18}$ In order to improve the electric-magnetic properties of $\mathrm{Gd}$ 123 in a wider field range, including low and high fields, we fabricated two types of large Gd-123 bulk materials using different starting powders by the in air processing.

\section{EXPERIMENT}

Two specimens were fabricated with the same nominal composition but different starting powders of $\mathrm{Gd}_{1.8} \mathrm{Ba}_{2.4} \mathrm{Cu}_{3.4} \mathrm{O}_{y}+0.2 \mathrm{BaCuO}_{2}$ and $\mathrm{GdBa}_{2} \mathrm{Cu}_{3} \mathrm{O}_{y}$ $+0.4 \mathrm{Gd}_{2} \mathrm{BaCuO}_{5}+0.2 \mathrm{BaCuO}_{2}$, which are denoted as $\mathrm{S} 1$ and $\mathrm{S} 2$, respectively. The Gd-123, Gd-211, and $\mathrm{BaCuO}_{2}$ powders were synthesized from high-purity $\mathrm{Gd}_{2} \mathrm{O}_{3}, \mathrm{BaCO}_{3}$, and $\mathrm{CuO}$ by solid-state reaction method, respectively. The precursor powder of sample $\mathrm{S} 1$ was prepared by mixing $\mathrm{Gd}_{2} \mathrm{O}_{3}$, $\mathrm{BaCO}_{3}, \mathrm{CuO}$, and $\mathrm{BaCuO}_{2}$ powders, while the precursor powder of $\mathrm{S} 2$ was prepared by mixing Gd-123, Gd-211, and $\mathrm{BaCuO}_{2}$ in a molar ratio of 1:0.4:0.2. The excessive $\mathrm{BaCuO}_{2}$ adding into the precursor powder of $\mathrm{S} 1$ was expected to suppress excessive $\mathrm{Gd}_{1-x} \mathrm{Ba}_{2+x} \mathrm{Cu}_{3} \mathrm{O}_{y}$. The $0.5 \mathrm{wt} \%$ Pt powder was added into the prepared precursor powders of $\mathrm{S} 1$ and $\mathrm{S} 2$ to refine the secondary phase. Then all the precursor powders were milled by a ball mill machine, respectively, to ensure that each powder was extremely homogenous. The wellmilled precursor powders were pressed into cylindrical pellets by a uniaxial die molding under a pressure of 15-20 MPa. Melt-textured $\mathrm{NdBa}_{2} \mathrm{Cu}_{3} \mathrm{O}_{y}$ (Nd-123) crystals with good quality as seeds were placed at the center of the 


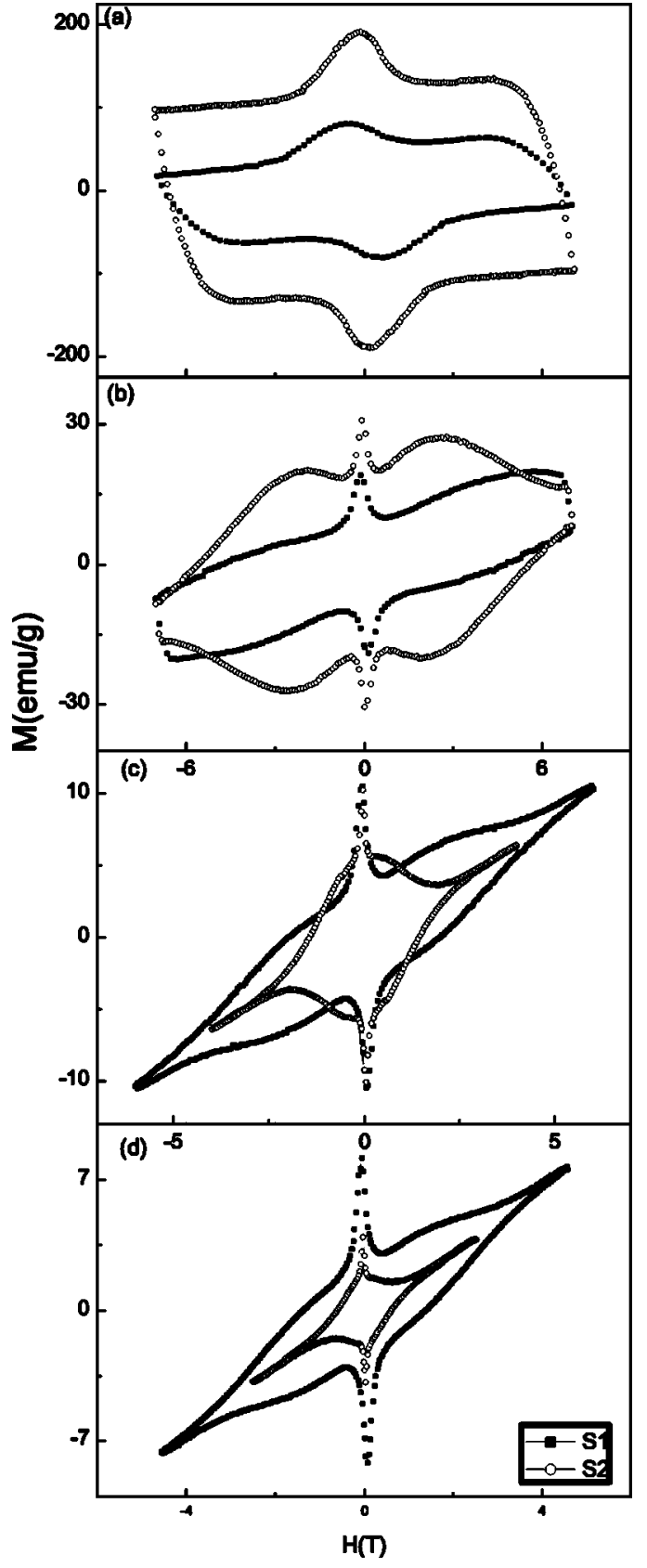

FIG. 1. Magnetic hysteresis loops of the samples (a) at $T=20 \mathrm{~K}$, (b): at $T$ $=60 \mathrm{~K}$, (c): at $T=74 \mathrm{~K}$, and (d) at $T=78 \mathrm{~K}$.

top surfaces of the $\mathrm{S} 1$ and $\mathrm{S} 2$ pellets before the melt processing started. $\mathrm{Al}_{2} \mathrm{O}_{3}$ plates were chosen as substrates during the domain growth. In order to avoid the loss of the liquid phase in the period of the solidification, $\mathrm{MgO}$ single-crystal plates were used to separate the samples from the $\mathrm{Al}_{2} \mathrm{O}_{3}$ substrates.

The melting process was performed in an isothermal box furnace in air. The samples were heated at a rate of $300{ }^{\circ} \mathrm{C} / \mathrm{h}$ to $1070{ }^{\circ} \mathrm{C}$ so as to melt partially, which is above the peritectic temperature of the Gd-123 phase but below the one of Nd-123. After a $0.5-1 \mathrm{~h}$ holding, the temperature decreased rapidly to about $1060{ }^{\circ} \mathrm{C}$, which is just above the peritectic transition temperature of Gd-123. Subsequently, the samples were cooled slowly to $1020{ }^{\circ} \mathrm{C}$ at a rate of $0.3-0.5{ }^{\circ} \mathrm{C} / \mathrm{h}$ and then cooled down to room temperature at a rate of $100{ }^{\circ} \mathrm{C} / \mathrm{h}$. The as-grown samples are nonsupercon-

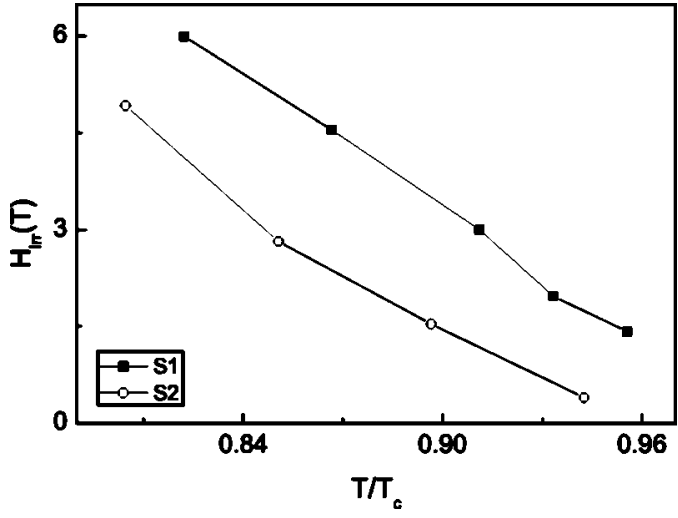

FIG. 2. The irreversibility fields of samples S1 and S2.

ducting owing to low oxygen content. Therefore, a postannealing in oxygen atmosphere is necessary. In this experiment, the samples were annealed at $400{ }^{\circ} \mathrm{C}$ for $50 \mathrm{~h}$ in flowing oxygen. In this way, samples with a single domain and with a diameter of $25 \mathrm{~mm}$ were fabricated successfully.

Two samples of $4 \times 2 \times 0.2 \mathrm{~mm}^{3}$ were cut from S1 and $\mathrm{S} 2$, respectively, to conduct a magnetic mesurement. The magnetization-temperature $(M-T)$ measurement at $10-\mathrm{Gs}$ field cooling showed that $T_{c}$ of samples S1 and S2 are 90 and $87 \mathrm{~K}$, respectively. Magnetic hysteresis loops at various temperatures and fields parallel to the $c$ axis up to $7 \mathrm{~T}$ were carried out by means of a commercial vibrating sample magnetometer. The field sweep rate was $d H / d t=0.12 \mathrm{~T} / \mathrm{min}$ in the measurements.

\section{RESULTS AND DISCUSSION}

The field dependence of magnetization $(M-H)$ for $\mathrm{S} 1$ and $\mathrm{S} 2$ at different temperatures are shown in Fig. 1. Because of $J_{c} \propto M$, shown in Fig. 1 are in fact the field dependence of $J_{c}$. It is seen that at $20 \mathrm{~K}$, the lowest temperature in our measurements, $J_{c}$ of $\mathrm{S} 2$ is higher than that of $\mathrm{S} 1$ for all the applied fields $(-7 \mathrm{~T} \leqslant H \leqslant 7 \mathrm{~T})$. This higher $J_{c}$ of $\mathrm{S} 2$ at low temperatures and fields maybe related to the fact that $\mathrm{S} 2$ has fine Gd-211 particles in its starting powder, which were the effective pinning centers. ${ }^{10,11}$ Whereas in S1 less Gd-211 might be on the precipice. Less Gd-211 phase means saving some $\mathrm{Gd}$ ions in $\mathrm{S} 1$. The saved $\mathrm{Gd}$ ions react with $\mathrm{BaCuO}_{2}$, forming $\mathrm{Gd}_{1+x} \mathrm{Ba}_{2-x} \mathrm{Cu}_{3} \mathrm{O}_{y}$ solid solution particles which are more effective pinning centers at higher temperatures and fields. This understanding is supported by the following fact. With elevated temperature, for example, at 60 and $74 \mathrm{~K}$ [see Figs. 1(b) and 1(c)], the $J_{c}$ of S2 is still larger than that of S1 only at low fields, whereas at the fields higher than a crossover field where $J_{c}$ of S2 is equal to that of S1, the $J_{c}$ of S1 is higher than that of S2. This crossover of the $J_{c}(H)$ relationship implies that the irreversibility field of $\mathrm{S} 1$ is higher than that of S2, as shown in Fig. 2. Because the temperature has been normalized with $T_{c}$, the higher $H_{\text {irr }}$ of S1 does not result from its higher $T_{c}$. When the temperature ascends further, say $T=78 \mathrm{~K}$ [see Fig. 1(d)], the $J_{c}$ of $\mathrm{S} 1$ is higher than that of $\mathrm{S} 2$ for the whole field (also $-7 \mathrm{~T} \leqslant H \leqslant 7 \mathrm{~T}$ ), supporting the explanation further. 


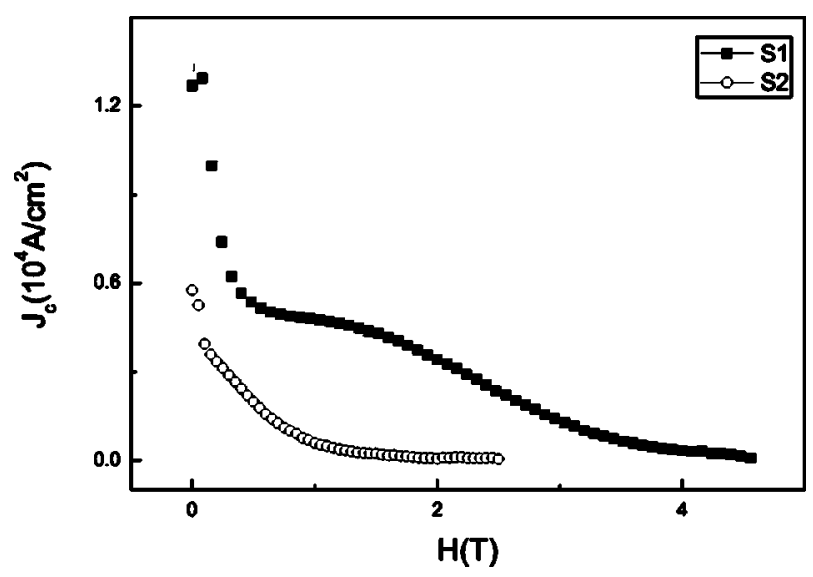

FIG. 3. The critical current densities vs applied magnetic field for S1 and S2 at $78 \mathrm{~K}$.

Now we compare the magnetic critical current densities of our samples, which are estimated by the well-known extended Bean critical state model. ${ }^{19}$ As an example, the $J_{c}-H$ curves at $78 \mathrm{~K}$ for S1 and S2 are shown in Fig. 3. The $J_{c}$ at zero magnetic field is of the same order of magnitude as reported. ${ }^{12-16}$ According to the above discussion, it is possible to further improve $J_{c}$ of MTG Gd-123 bulk at different fields by properly controlling their starting powders, although more work is needed.

\section{CONCLUSIONS}

In summary, cylindrical single grains of $\mathrm{GdBa}_{2} \mathrm{Cu}_{3} \mathrm{O}_{y}$ with a diameter of $25 \mathrm{~mm}$ were successfully fabricated in air in terms of different starting powders. The measurements showed that the starting powders influence the $J_{c}-H$ curve apparently. The sample with the Gd-211 addition in the starting powder has higher $J_{c}$ at low temperatures and fields. On the other hand, the sample fabricated using $\mathrm{Gd}_{1.8} \mathrm{Ba}_{2.4} \mathrm{Cu}_{3.4} \mathrm{O}_{y}+0.2 \mathrm{BaCuO}_{2}$ as starting powders has higher $J_{c}$ at high temperatures and fields. The results sug- gested that it was possible to obtain a large Gd-123 single grain with high $J_{c}$ by properly controlling the staring powder gradients by a simpler and cheaper process.

\section{ACKNOWLEDGMENTS}

This work was supported by the Ministry of Science and Technology of China (Grant No. G1999064602), National Nature Science foundation of China (NNSFC, Grant No. 19994016), and Open Foundation of Testing of Nanjing University (Grant No. 0204001308).

${ }^{1}$ S. Jin, T. H. Tiefel, R. C. Sherwood, M. E. Davis, R. B. van Dover, G. W. Kammiott, R. A. Fastnacht, and H. D. Keith, Appl. Phys. Lett. 52, 2074 (1988).

${ }^{2}$ K. Salama, V. Selvamanickam, L. Gao, and K. Sun, Appl. Phys. Lett. 54, 2352 (1989).

${ }^{3}$ M. Murakami, S. Gotoh, H. Fujinoto, K. Yamaguchi, N. Koshizuka, and S. Tanaka, Supercond. Sci. Technol. 4, S49 (2000).

${ }^{4}$ L. Zhou, P. Zhang, P. Ji, K. Wang, J. Wang, and X. Wu, Supercond. Sci. Technol. 3, 490 (1990).

${ }^{5}$ A. Erb, J. Y. Genoud, F. Marti, M. Daumling, E. Walker, and R. Flukiger, Low Temp. Phys. 105, 1023 (1996).

${ }^{6}$ A. A. Zhukov et al., Phys. Rev. B 51, 12704 (1995).

${ }^{7}$ H. Kupfer, T. Wolf, C. Lessing, A. A. Zhukov, R. Meier-Hirmer, W. Schauer, and H. Wuhl, Phys. Rev. B 58, 2886 (1998).

${ }^{8}$ M. Muralidhar, N. Sakai, N. Chikumoto, M. Jirsa, T. Machi, M. Nishiyama, Y. Wu, and M. Murakami, Phys. Rev. Lett. 89, 237001 (2002).

${ }^{9}$ M. R. Koblischka and M. Murakami, Supercond. Sci. Technol. 13, 738 (2000).

${ }^{10}$ M. Murakami, N. Sakai, T. Higuchi, and S. I. Yoo, Supercond. Sci. Technol. 9, 82 (1996).

${ }^{11}$ M. Muralidhar, M. Jirsa, N. Sakai, and M. Murakami, Supercond. Sci. Technol. 16, R1 (2003).

${ }^{12}$ J. Q. Dai, Z. X. Zhao, and A. Hu, Physica C 406, 63 (2004).

${ }^{13}$ M. Muralidhar, M. Jirsa, N. Sakai, and M. Murakami, Physica C 392396, 619 (2003).

${ }^{14}$ E. Sudhakar Reddy and T. Rajasekharan, Physica C 316, 279 (1999).

${ }^{15}$ S. Nariki, N. Sakai, and M. Murakami, Physica C 357-360, 629 (2001).

${ }^{16}$ S. Nariki, H. Hinai, N. Sakai, M. Murakami, and M. Otsuka, Physica C 357-360, 817 (2001).

${ }^{17}$ K. Rogacki, Phys. Rev. B 68, 100507 (2003).

${ }^{18}$ J. Roa-Rojas, R. Menegotto Costa, P. Pureur, and P. Prieto, Phys. Rev. B 61, 12457 (2000).

${ }^{19}$ D. X. Chen and R. B. Goldfarb, J. Appl. Phys. 66, 2489 (1989). 